\title{
STATE-ORIENTED MAINTENANCE STRATEGY FOR DETERIORATING SEGMENTAL LINING OF TUNNEL
}

\author{
Qing $\mathrm{AI}^{1}$, Yong YUAN $2,3^{*}$ \\ ${ }^{1}$ Department of Civil Engineering, Shanghai Jiao Tong University, Shanghai, China \\ ${ }^{2}$ College of Civil Engineering, Tongji University, Shanghai, China \\ ${ }^{3}$ State Key Laboratory of Disaster Reduction in Civil Engineering, Tongji University, Shanghai, China
}

Received 27 April 2018; accepted 29 June 2018

\begin{abstract}
The lining of tunnel is the principal part in ensuring its usage for transportation or utilities. Maintenance during operation is the way to keep structure in the state of high performance. To consider the efficiency of maintenance within limited resources (maintenance cost), state-oriented maintenance (SOM) approach is proposed. The SOM approach takes service state of the tunnel lining as a levelled target which should be maintained by different degrees of repairing measures. The evolution of service state of tunnel lining is modelled as a non-stationary Gamma process in this investigation, as this process can characteristic the variations of degradation rates. Two cases of numerical analysis with SOM approach give their lifetime maintenance costs. Case one (MS-1) sets SOM strategy with two-levelled service state. The other case (MS-2) adopts SOM strategy with multi-levelled service state. The numerical results demonstrate that the later one would reduce maintenance cost during the whole service life of tunnel lining.
\end{abstract}

Keywords: tunnel, maintenance, structure, service state, state-oriented maintenance.

\section{Introduction}

Tunnels constructed in urban cites, such as metro system or underground roads, are important infrastructures relating to people's life security. The lining of tunnel is the principal part in ensuring its usage for transportation or utilities, so it requires high-level safety and excellent performance during its whole service life. However, many surveys on tunnel linings show that they deteriorated in their early ages of service life, of which several severe deteriorations have happened and caused accidents in the past decades (Yuan et al. 2012). In order to maintain the service performance of tunnel structure, repairing or inspection activities are required to be scientific planned and carried out. On the other hand, the repair and inspection work of tunnel lining is very heavy, but the maintenance budget is limit. Thus, the maintenance is becoming a stressing job for the operating company of tunnel.

Maintenance strategy is the method of allocating maintenance resources to improve maintenance effects, and it is becoming more important in current situation. Research on maintenance strategy is booming in recent years (Do et al. 2015; Ponchet et al. 2012), and the maintenance strategy of tunnel begins to attract people's atten- tion (Baji et al. 2017; Wang et al. 2017). In the framework of maintenance strategy, usually a non-periodic inspection policy is proposed to establish the correspondence function between degradation indicator and inspection interval, and the policy will be optimized base on a predictive model in lifetime maintenance. This non-periodic inspection policy has been both applied to one-unit degradation system (Grall et al. 2002) and two-unit degradation system (Castanier et al. 2005). Also, the periodic inspection policy (Neves et al. 2011) and multi-variable degradation systems (Barker, Newby 2009) are studied in other kinds of maintenance strategies. However, current researches have paid a lot of attention on inspection policy, but there is less study on the criterion of repairing action. Also, repeated shut down or failure are allowed in above maintenance strategies (Jardine et al. 2006), which is impracticable and rarely happened in civil engineering structures. Thus, directly extrapolate the above maintenance strategies to civil engineering structure is unsuitable.

Usually the service states are only classified as normal and failure, which represents the view in industrial engineering. But for the civil engineering structures, limit

${ }^{*}$ Corresponding author. E-mail: yuany@tongji.edu.cn 


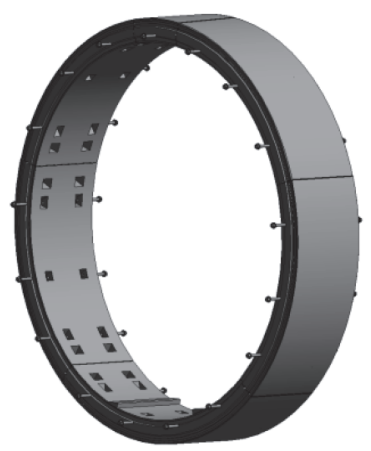

(a) One ring of tunnel lining

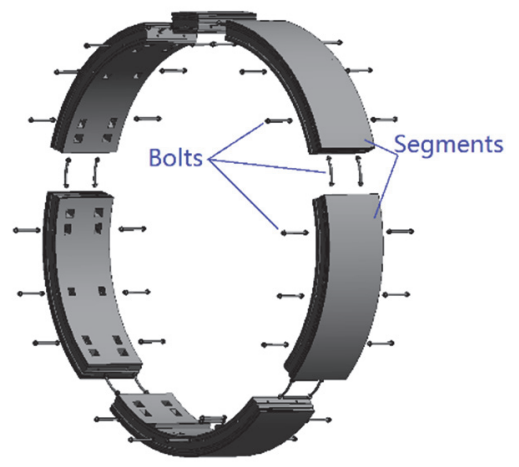

(b) Components of one ring of tunnel lining

Figure 1. One ring of tunnel lining

states are distinguished as ultimate and serviceability. In order to demonstrate the progressive degradation of structural performance, the service states can be divided into several levels (Yuan et al. 2012; Kavussi et al. 2017). The levelled service states demonstrate the degradation process of civil engineering structure, which is an extension of the concept of limit states. Also, the repairing measures for structures can be classified into different degrees according to their intensity and cost. Thus, how to choose repairing measures for different levels of service states is worth studying. In maintenance practice, the arrangement of repairing actions during the service life will also affect maintenance costs and effectiveness.

State-oriented maintenance (SOM) approach is proposed in this paper. The SOM approach takes service state of the structure as a levelled target which should be maintained by different degrees of repairing measures. The evolution of service state of tunnel lining is modelled as non-stationary Gamma process in this investigation, as this process can characteristic the variation of degradation rate. Two cases of numerical analysis with SOM approach give their lifetime maintenance costs, which represent two different classifications of service states. Case one (MS-1) sets SOM strategy with two-levelled service state. The other case (MS-2) adopts SOM strategy with multi-levelled service state. Finally, discussions and conclusions of SOM approach are drawn from the results of numerical investigation.

\section{State-oriented maintenance (SOM)}

\subsection{Assessment of service state}

Different from the commonly used degradation indicator in maintenance strategy, service state comprehensively represents the service performance of structure other than one aspect. At present, the assessment of service state of tunnel lining usually adopts analytic hierarchy process (AHP) method (JTG H12-2015 2015). Determining the weight of different indicators is an important step for adopting this method, many scholars have conducted research in this area. For example, Li et al. (2017) proposed a regression method to get the weight of different defects from expert opinions in assessing the service condition of metro shield tunnel. Fuzzy method and AHP evaluation procedure are also applied to the health evaluation of structure (Zhang et al. 2014; Chen et al. 2017; Lyu et al. 2018). The differences between their studies are the division of hierarchical structure and estimation methods of weight index, but they all adopt a systematic evaluation method and integrate multiple indicators to comprehensively reflect the service performance of structure.

Assessment of service state of tunnel can be completed by two steps: (1) calculating the index of service state; (2) categorize it into different service states by predefined threshold values of different service states. The whole tunnel structure can be divided into many rings of tunnel lining, and one ring of tunnel lining consists of segments, sealants and bolts, as shown in Figure 1. Thus, the index of service state of whole tunnel structure can be calculated by Eqn (1) with considering inspection time:

$$
X(t)=\sum_{i=1}^{n} x_{i}(t) \times \frac{w_{i}}{\sum_{i=1}^{n} w_{i}} .
$$

In Eqn (1), the $w_{i}$ is the weight of one ring of tunnel lining, $x_{i}(t)$ is the service state index of one ring of tunnel lining at inspection time, and $X(t)$ is the service state index of whole tunnel structure. The assessment of one ring of tunnel lining is similar, just calculating the product of the score and weight of all belonging components. It should be noted that the assessment of one ring of tunnel lining generally subject to the worst component, as shown in Eqn (2), in which $x_{i j}(t)$ is the score of one component belonging to the ring of tunnel lining:

$$
x_{i}(t)=\max \left(x_{i j}(t)\right) \text {. }
$$

There are different methods of classifying the service state of tunnel. One method is just setting one threshold to distinguish the normal state and failure state, which is call two-levelled service state. This ordinary method is widely used in current maintenance strategies. Another method considers different kinds of service states of structure. By setting several thresholds according to degradation process of structure, the service states of structure can be 
Table 1. Multi-levelled service state of structure

\begin{tabular}{|l|l|l|}
\hline $\begin{array}{c}\text { Service } \\
\text { state }\end{array}$ & $\begin{array}{c}\text { Index of } \\
\text { service state }\end{array}$ & \\
\hline Level I & $X(t)<S_{1}$ & Normal. The service performance is good. \\
\hline Level II & $S_{1} \leq X(t)<S_{2}$ & $\begin{array}{l}\text { Slightly deteriorated. The service performance start deterioration, but this does not affect normal } \\
\text { usage and functionality. }\end{array}$ \\
\hline Level III & $S_{2} \leq X(t)<S_{3}$ & $\begin{array}{l}\text { Moderately deteriorated. The deterioration of service performance starts affecting normal usage and } \\
\text { functionality. }\end{array}$ \\
\hline Level IV & $S_{3} \leq X(t)<S_{4}$ & $\begin{array}{l}\text { Seriously deteriorated. The deterioration of service performance is severe, most normal usages and } \\
\text { functionalities of structure are affected but will not lead to safety problems. }\end{array}$ \\
\hline Level V & $X(t) \geq S_{4}$ & Dangerous. The structure has safety problem, and it should be abandoned or rehabilitated. \\
\hline
\end{tabular}

classified into multiple levels. This method can be called multi-levelled service state.

In the progressively degradation of tunnel, it is supposed that the durability of structure is firstly affected, then the feasibility of structure is interfered, and finally is the arising of safety problem. Thus, $X(t)$ can be categorized into 5 levels from the view of multi-levelled service state, which are: normal (Level I, $X(t)<S_{1}$, slightly deteriorated (Level II, $S_{1} \leq X(t)<S_{2}$ ), moderately deteriorated (Level III, $S_{2} \leq X(t)<S_{3}$ ), seriously deteriorated (Level IV, $S_{3} \leq X(t)<S_{4}$, dangerous (Level V, $X(t) \geq S_{4}$ ). The descriptions and definitions of multi-levelled service state of structure are shown in Table 1.

It should be noted that deterioration will progressively affect the performance of structure, and it is reflected on the development of index of service state $X(t)$. In Table 1, reaching threshold $S_{2}$ means that the structure is going to lose its durability, reaching threshold $S_{3}$ means that the structure is going to lose its feasibility and reaching threshold $S_{4}$ means that the structure is going to lose its safety.

\subsection{State-oriented maintenance approach}

The state-oriented maintenance (SOM) approach takes the levels of service state as the criteria of planning different degrees of repairing measures. As presented in previous section, there are two kinds of viewpoints in classifying the service states of structure. One kind of view thinks that the service states should be divided more delicately so that the implementation of maintenance activities could be more refined. Another kind of view considers two service states (safe and failure) of structure. The proposed SOM approach can both deal with the two viewpoints.

SOM approach with multi-levelled service state is shown in Figure 2, where multiple service states and multiple repairing measures are mapped with each other. In this situation, service state index $X(t)$ decides which level this structure should be assessed, then the corresponding repairing measure is prescribed according to SOM approach.

Similarly, SOM approach with two-levelled service state is rather simple, the service state can only be assessed

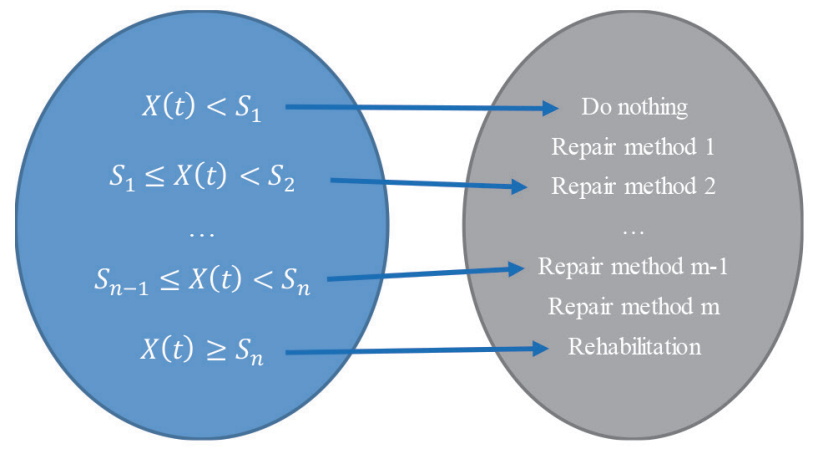

Figure 2. SOM approach with multi-levelled service state

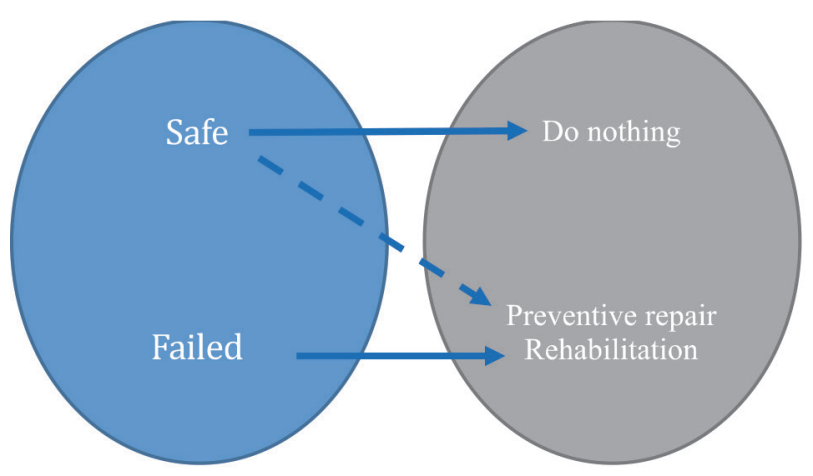

Figure 3. SOM approach with two-levelled service state

as safe or failed through a threshold value. In corrective maintenance, the repair actions are only carried out when the structure is failed. In preventive maintenance, repair action will preventively conduct before the structure is going to fail, as shown in Figure 3.

The difference of SOM approach dealing with the two viewpoints is that there are more degrees of repairing measures for multi-levelled service state. In order to better illustrate SOM approach, more specific examples of SOM approach dealing with a multi-levelled service state and a two-levelled service state are compared in Table 2. In multi-levelled service state, the repairing measures are categorized into different degrees (minor repair, medium repair and major repair) according to their effects and costs. 
Table 2. SOM approach dealing with different classifications of service state

\begin{tabular}{|c|c|c|c|}
\hline \multicolumn{2}{|c|}{ Multi-levelled service state } & \multicolumn{2}{|c|}{ Two-levelled service state } \\
\hline Service state & Repairing measures & Service state & Repairing measures \\
\hline Level I $\left(X(t)<S_{1}\right)$ & Do nothing & \multirow{3}{*}{ Safe $(X(t)<S)$} & \multirow{3}{*}{ Do nothing } \\
\hline Level II $\left(S_{1} \leq X(t)<S_{2}\right)$ & Minor repair & & \\
\hline Level III $\left(S_{2} \leq X(t)<S_{3}\right)$ & Medium repair & & \\
\hline Level IV $\left(S_{3} \leq X(t)<S_{4}\right)$ & Major repair & Safe $(S \leq X(t)<F)$ & Preventive repair (= Major repair) \\
\hline Level V $\left(X(t) \geq S_{4}\right)$ & Rehabilitation & Failed $(X(t) \geq F)$ & Rehabilitation \\
\hline
\end{tabular}

The purpose of proposing SOM approach is to optimize maintenance process and improve the efficiency of repairing activities. In order to implement SOM approach to tunnel lining, two problems must be solved, which are the modelling of evolution of service state considering the variation of degradation rate and the effects of repairing activities in degradation model.

\section{Modelling the degradation process}

In SOM approach, modelling the degradation process is necessary, because the optimization of maintenance strategy has to be conducted base on the lifetime prediction of degradation process. The stochastic model is recommended for simulating the degradation of structures subjected to gradual deterioration (Nicolai et al. 2007; Zambon et al. 2017). Generally, it is reasonable to assume that the deterioration level of tunnel structure will gradually become severe with time if no repairing activity is applied, and it can be regarded as a monotonic process. The characteristic of a gamma process is that it is strictly monotonically increasing with independent and non-negative increments having a Gamma distribution with identical scale parameters (Van Noortwijk 2009; Strauss et al. 2017). Thus, we propose the Gamma process as the stochastic model for simulating the degradation process of tunnel lining.

\subsection{Modelling the variation of degradation rate}

\subsubsection{Non-stationary Gamma process}

The following describes a stationary Gamma process. Assuming a random quantity $X$ has a Gamma distribution with shape parameter $\alpha>0$ and scale parameter $\beta>0$, and its probability density function is given by:

$$
G a(x \mid \alpha, \beta)=\frac{x^{\alpha-1} \beta^{\alpha}}{\Gamma(\alpha)} \exp (-\beta x) I_{(0, \infty)}(x)
$$

where $I_{(0, \infty)}(x)=1$ for $x \in(0, \infty)$ and $I_{(0, \infty)}(x)=0$ for $x \notin(0, \infty)$, and $\Gamma(\alpha)=\int_{z=0}^{\infty} z^{\alpha-1} e^{-z} d z$ is the Gamma function for $\alpha>0$. Furthermore, let $X(t)$ be the index of service state of the structure at time $t$, and its degradation is a Gamma process with shape parameter $\alpha$ and scale parameter $\beta$. Then this Gamma degradation process has the following properties:

1. $X(0)=0$ with probability of one;

2. $X(t)-X(s) \sim G a(\alpha(t-s), \beta)$ for all $t>s \geq 0$;

3. $X(t)$ has independent increment.

The probability density function (PDF), expectation and variance at time $t$ are given by:

$$
\begin{aligned}
& f_{X(t)}(x)=G a(x \mid \alpha t, \beta) ; \\
& E(X(t))=\frac{\alpha t}{\beta} ; \\
& \operatorname{Var}(X(t))=\frac{\alpha t}{\beta^{2}} .
\end{aligned}
$$

The cumulative distribution function (CDF) of the first passage time can be easily derived from the probability distribution of $X(t)$. The CDF of the Gamma process $X(t)$ exceeding a level $z>0$ is represented by $F_{G a}(t, z)$, which can be written as:

$F_{G a}(t, z)=P(X(t) \geq z)=\int_{x=z}^{\infty} f_{X(t)}(x) d x=\frac{\Gamma(\alpha t, z \beta)}{\Gamma(\alpha t)}$,

where $\Gamma(\alpha, x)=\int_{z=x}^{\infty} z^{\alpha-1} e^{-z} d z$ is the Gamma function for all $x \geq 0$ and $\alpha>0$. The advantage of modeling degradation process through Gamma process is that the required mathematical calculations are relatively straightforward.

However, the degradation process may accelerate or decelerate with time due to different conditions during the service life of structure. Thus, the evolution of service state of tunnel structure does not strictly follow a stationary Gamma process. A time-transformation method is proposed to model non-stationary Gamma process (Nicolai et al. 2007). Thus, this mathematical transformation can be used to characteristic the variation of degradation rate of tunnel structure.

For example, the time is transformed to a variable via a non-decreasing function $v=v(t)$, to obtain a nonstationary degradation process. There are several time- 
transformation functions available, such as linear function $v=k t$, exponential transformation $v=1-\exp \left(-\lambda \cdot t^{\gamma}\right)$ and power transformation $v=t^{q}$. It is easy to derive that the increment of a non-stationary Gamma process $X(t)$ between $t$ and $s$ is:

$$
X(t)-X(s) \sim G a(v(t)-v(s), \beta), t>s \geq 0 .
$$

The PDF of $X(t)$ is:

$$
f_{X(t)}(x)=G a(x \mid v(t), \beta)
$$

and the CDF of the first passage time for a non-stationary Gamma process $X(t)$ exceeding level $z>0$ is

$$
\begin{aligned}
F_{G a}(t, z)= & P(X(t) \geq z)= \\
& \int_{x=z}^{\infty} f_{X(t)}(x) d x=\frac{\Gamma(v(t), z \beta)}{\Gamma(v(t))},
\end{aligned}
$$

where $\Gamma(\alpha, x)=\int_{z=x}^{\infty} z^{\alpha-1} e^{-z} d z$ is the Gamma function for all $x \geq 0$ and $\alpha>0$ and $\Gamma(\alpha)=\int_{z=0}^{\infty} z^{\alpha-1} e^{-z} d z$ is the
Gamma function for $\alpha>0$.

Considering a power transformation function $v=t^{q}$, the simulation of a non-stationary Gamma process is shown in Figure 4. It shows the realizations of a non-stationary Gamma processes with shape function $v=t^{0.5}$ and scale parameter $\beta=0.2$.

\subsubsection{Parameter estimation}

In order to apply the Gamma process to practical modeling of tunnel structure, the commonly used maximum likelihood method is applied in this research. Consider a non-stationary Gamma process $X(t)$ with shape function $v(t)=c t^{b}$ and scale parameter $\beta$. Assume that there is a data set available consisting of $n$ inspection times $t_{i}, i=1, \ldots, n$, where $0=t_{1}<t_{2}<\ldots t_{n}$, and the corresponding observations of the cumulative amount of degradation $x_{i}, i=1, \ldots, n$, where $0=x_{1}<x_{2}<\ldots<x_{n}$. The parameters $c, b$ and $\beta$ of the Gamma process can be estimated by maximizing the likelihood function of the independent increments of the degradation level with respect to $c, b$ and $\beta$ (Nicolai et al. 2007; Van Noortwijk 2009). The likelihood function of the observed degradation increment $y_{i}=x_{i}-x_{i-1}, i=1, \ldots, n$, is a product of independent Gamma densities:

$$
\prod_{i=1}^{n} f_{X\left(t_{i}\right)-X\left(t_{i-1}\right)}\left(y_{i}\right)=\prod_{i=1}^{n} G a\left(y_{i} \mid c t_{i}^{b}-c t_{i-1}^{b}, \beta\right) .
$$

Assuming independent observations and given the maximum likelihood estimators of parameters in Gamma process, the structural degradation of metro tunnel can be predicted via Eqn (3).

\subsection{Modelling the effects of repairing activities}

The repairing activities for structure can be categorized into imperfect maintenance according the concepts in

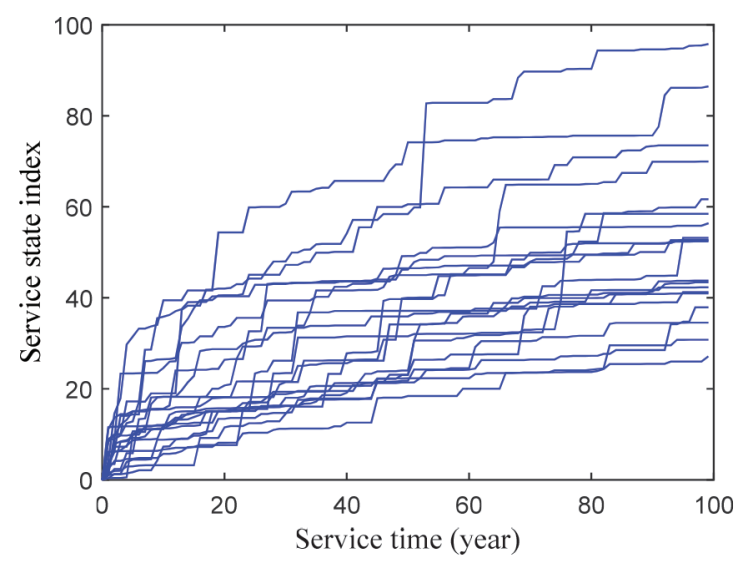

Figure 4. Simulating the variation of degradation rate by non-stationary Gamma processes

industrial engineering, which is different from perfect maintenance (Nicolai et al. 2009). Perfect maintenance is the service state goes back to its initial state (as good as new) after maintenance/repairing. Assuming the service state index before maintenance is $X(t)^{-}(\geq \xi$, in which $\xi$ is the threshold value of repairing) at time $t$, the perfect maintenance means the service state index after maintenance is $X(t)^{+}=0$. However, imperfect maintenance means that the service state of structure cannot be changed to its initial state after maintenance, the service state index after imperfect maintenance $X(t)^{+}$is a value between 0 and $X(t)^{-}$. Considering as imperfect maintenance, repairing activities can be classified into different degrees based on their effects of improving service state of structure.

The repairing measures commonly used in Shanghai Metro are:

1. Aramid fiber reinforcement plastics (AFRP);

2. Steel plate bond reinforcement (as shown in Figure 5);

3. Grouting reinforcement of foundation.

They mainly have two kinds of effects:

1. Reducing degradation rate;

2. Reducing service state index, which reduce the level of service state indirectly.

In practice, the repairing measures may reduce service state index and degradation rate at the same time, but in this research the two kinds of effects are considered as individual for simplicity. Referring to Table 2, usually the major and medium repair are designed to significantly improve the service performance of structure, thus they are suitable to be modelled as reduce service state index. The minor repair has limit effect on improving service performance of structure, and it is suitable to be modelled as the reduction of degradation rate.

\subsubsection{Reducing service state index}

Assuming $A(t)$ is the effect of maintenance measure, $X(t)^{-}$is the service state index of structure before maintenance, and $X(t)^{+}$is the service state index after maintenance. Thus, the reduction of service state index is: 


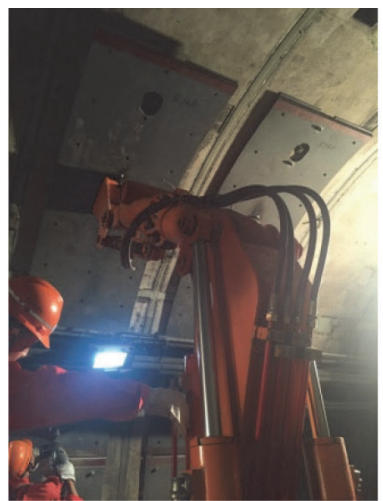

(a) In implementation

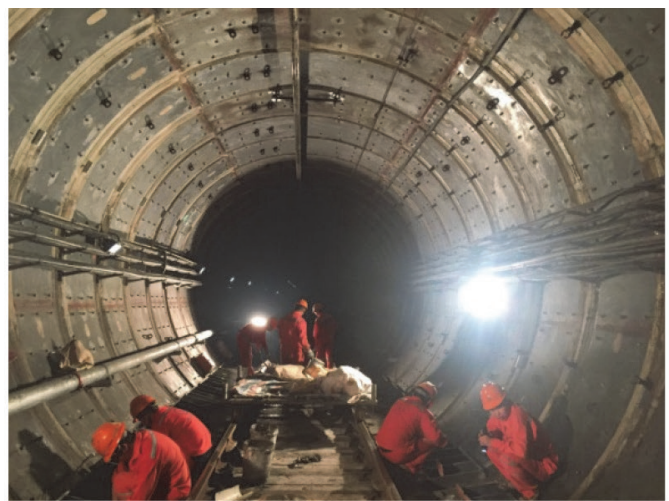

(b) After implementation

Figure 5. Implementing steel plate bond reinforcement for metro tunnels

$$
A(t)=X(t)^{-}-X(t)^{+}
$$

Considering the accuracy of repairing activities is not high, the value of $A(t)$ can be assumed as a random value. Assuming $A(t)$ is random value follows uniform distribution, as shown in Eqn (13), which $a=0, b=X(t)^{-}$. It is expressed as:

$$
A(t) \sim U(a, b) .
$$

Thus, after implementing repairing activity, the effect is reflected as a random reduction of service state index (means the service state improved) on the degradation process.

\subsubsection{Reducing degradation rate}

In addition to the effect of reducing service state index, the imperfect maintenance may also change the degradation rate of structure. For example, some repair measures may enhance the resistance of structure, therefore the degradation rate in the follow-up deterioration process will slowdown, which also achieved the purpose of reducing risk.

In Gamma degradation process, the reduction of degradation rate can be simulated by changing the shape parameter of the Gamma process (Do et al. 2015). However, the degradation rate cannot be reduced without limitation. After several identical repairs, the maintenance effect will remain unchanged. Assuming $\alpha$ is the shape parameter of the Gamma process before applying maintenance measure, and $d[i]$ is the effect of the $i_{\text {th }}$ repair, which is a coef- ficient between 0 and 1 . The new shape parameter $\alpha^{*}$ after applying $i_{\text {th }}$ maintenance measure can be modeled by:

$$
\alpha^{*}=\alpha \times d[i] \text {. }
$$

In numerical modelling, this intervention leads the degradation to a more smoothly developed process, which delays the arriving time of ultimate service states.

\section{Formation of the problem}

\subsection{Problem description}

In this study, SOM approach dealing with different classifications of service state will be investigated. Multi-levelled service state represents a refine maintenance method; otherwise the two-levelled service state represents an extensive maintenance method. The two methods adopting SOM approach cooperated with periodic inspection policies constitute two maintenance strategies, which are MS-1 and MS-2. Their effects are compared through numerical simulation of a real tunnel in the light of lifetime maintenance cost under safety requirement.

The details of the two maintenance strategies are shown in Table 3. It should be noted that the assumptions of different degrees of repairing measures in SOM approach refer to Section 2.2, and the preventive repair in two-levelled service state is the same with major repair in multi-levelled service state.

In order to get the minimum lifetime maintenance cost of different maintenance strategies under safety requirement, the parameters of the two maintenance strate-

Table 3. Parameters to be optimized in different maintenance strategies

\begin{tabular}{|l|l|l|}
\hline \multicolumn{1}{|c|}{ Maintenance strategy No. } & \multicolumn{1}{|c|}{ MS-1 } & \multicolumn{1}{c|}{ MS-2 } \\
\hline \multicolumn{1}{|c|}{ Classification of service states } & \multicolumn{1}{|c|}{ Multi-levelled service state } & Two-levelled service state \\
\hline Parameters already known & $S_{4}$ & $F$ \\
\hline Parameters to be optimized & $\begin{array}{l}\text { Maintenance thresholds: } S_{1}, S_{2}, S_{3} \\
\text { Inspection interval: } \tau\end{array}$ & $\begin{array}{l}\text { Maintenance threshold: } S \\
\text { Inspection interval: } \tau\end{array}$ \\
\hline
\end{tabular}


gies should be optimized at the same time. In general, the threshold for whether a structure enters a dangerous state (failure) is known and can be learned from previous experiments or calculations. Therefore, the parameters that need to be optimized in the two maintenance strategies are inspection interval and maintenance thresholds, as shown in Table 3.

\subsection{Mathematical formulation}

The proposing problem involves non-stationary Gamma process and imperfect maintenance modelling, which is difficult to apply the theoretical formulas in the calculation of lifetime maintenance cost in this case (Van, Bérenguer 2012). In this study, we propose a simulation-based method to calculate the expected number of repairing actions and failures during the service life of tunnel structure. The lifetime maintenance cost can be computed by:

$$
C_{\text {lifetime }}=C_{\text {insp }} \cdot E\left(N_{\text {insp }}\right)+\sum_{i=1}^{m} C_{r_{i}} \cdot E\left(N_{r_{i}}\right)+C_{f} \cdot E\left(N_{f}\right)
$$

where $C_{\text {lifetime }}$ is the lifetime maintenance cost during service life of tunnel structure. $N_{i n s p}$ is the number of inspection during service life, and $C_{\text {insp }}$ is the cost per inspection. Assuming there are $m$ types repairing methods of the tunnel. $N_{r_{i}}$ is the number of repairing measures of type $i$ applied during the service life of tunnel structure, and $C_{r_{i}}$ is the cost of maintenance type $i . C_{f}$ is the cost of rehabilitation when a failure occurs, and $N_{f}$ is the number of failures occurred. $E(\cdot)$ is the expectation operator.

One constraint of the optimization is that the probability of failure during the service life should be lower than a threshold level. The probability of failure of tunnel structure during service life can be estimated from $E\left(N_{f}\right)$ if the numbers of simulations are large enough. From the standards of tunnel structure, the value of probability of failure should be less than $1.1 \times 10^{-4}$ in the lifetime of tunnel (GB50153-2008 2008).

From the above, the mathematical formulation of the optimization problem is given by:

$$
\begin{gathered}
\min _{M S-1, M S-2}\left\{C_{\text {lifetime }}\right\} \\
\text { s.t. } P_{f}<1.1 \times 10^{-4}, \\
\text { where } M S-1=\left\{S_{1}, S_{2}, S_{3}, \tau\right\} ; \\
M S-2=\{S, \tau\} .
\end{gathered}
$$

\subsection{Optimization algorithm}

There are various methods for solving the maintenance and rehabilitation optimization of large infrastructure systems (Farran, Zayed 2015; Liu, Madanat 2015; Marzouk, Omar 2013; Zhu et al. 2015; Jin et al. 2016, 2017; Yin et al. 2018). The optimization algorithm employed in SOM approach is the nested enumeration algorithm. Take MS- 1 as an example, assume $k$ denotes the parameters in maintenance strategy, $n$ is the number of simulations of the degradation model, and $t$ is the service time of tun- nel structure in one simulation. The algorithm will firstly check the condition of the system at every time step, and a failure will be recorded if the condition indicator exceeds $S_{4}$ (it is $F$ in MS-2). Otherwise if the system is safe, the algorithm will check whether it's time to conduct an inspection. Corresponding types of repairing measures will apply if the inspected condition indicator exceeds $S_{i}$ (it is $S$ in MS-2), and the next inspection time is also scheduled based on a periodic inspection plan. Numbers of failure, inspection and repair are calculated in each lifetime simulation with different maintenance strategy parameters. At last, the lifetime maintenance costs with different parameters are calculated by Eqn (15), and the minimum maintenance cost and its strategy parameters fulfill the requirement of structural safety is chosen as the optimal ones.

The procedure of the nested enumeration algorithm for SOM approach is presented in Figure 6, which takes MS-1 as an example.

\section{Numerical investigation}

\subsection{Degradation modelling of investigated tunnel}

The SOM approach is investigated with tunnel structures of Shanghai Metro Line 1. The investigated tunnel is the interval tunnel from South Huangpi Road Station to People Square Station, which is constructed by shield tunneling method and put into operation in 1995 . The outer radius of the tunnel segmental ring is $6.2 \mathrm{~m}$, and the inner radius is $5.5 \mathrm{~m}$. The tunnel is buried in soft soil areas, consisting of mainly silty clay or mucky clay with very weak performance.

As the service time of tunnel structure increases, the deterioration of structural performance is observed. Among the different kinds of defects in metro tunnel, the convergence is the most representative and critical one. It is usually considered to be the conclusive indicator in assessing the service condition of tunnels (Ai et al. 2016). To simplify the hierarchical analysis process of service state assessment, the convergence is used as the indicator of service state of investigated tunnel in this numerical investigation. It should be noted that in the practical implementation of SOM approach, the service state of structure should be assessed comprehensively, from aspects including safety, serviceability, durability and so on.

The previously proposed non-stationary Gamma process with power time transformation is applied to simulate the degradation process of investigated tunnel. Assume the time transformation function of shape parameter in the non-stationary Gamma process as $c t^{q}$, and the scale

Table 4. Statistics of the inspection data

\begin{tabular}{|l|c|c|}
\hline \multicolumn{1}{|c|}{ Time period (years) } & $t_{1}-t_{0}=1.82$ & $t_{2}-t_{1}=7$ \\
\hline Mean $(\mathrm{mm})$ & 9.1593 & 25.6533 \\
\hline Standard deviation $(\mathrm{mm})$ & 3.5078 & 13.0449 \\
\hline
\end{tabular}




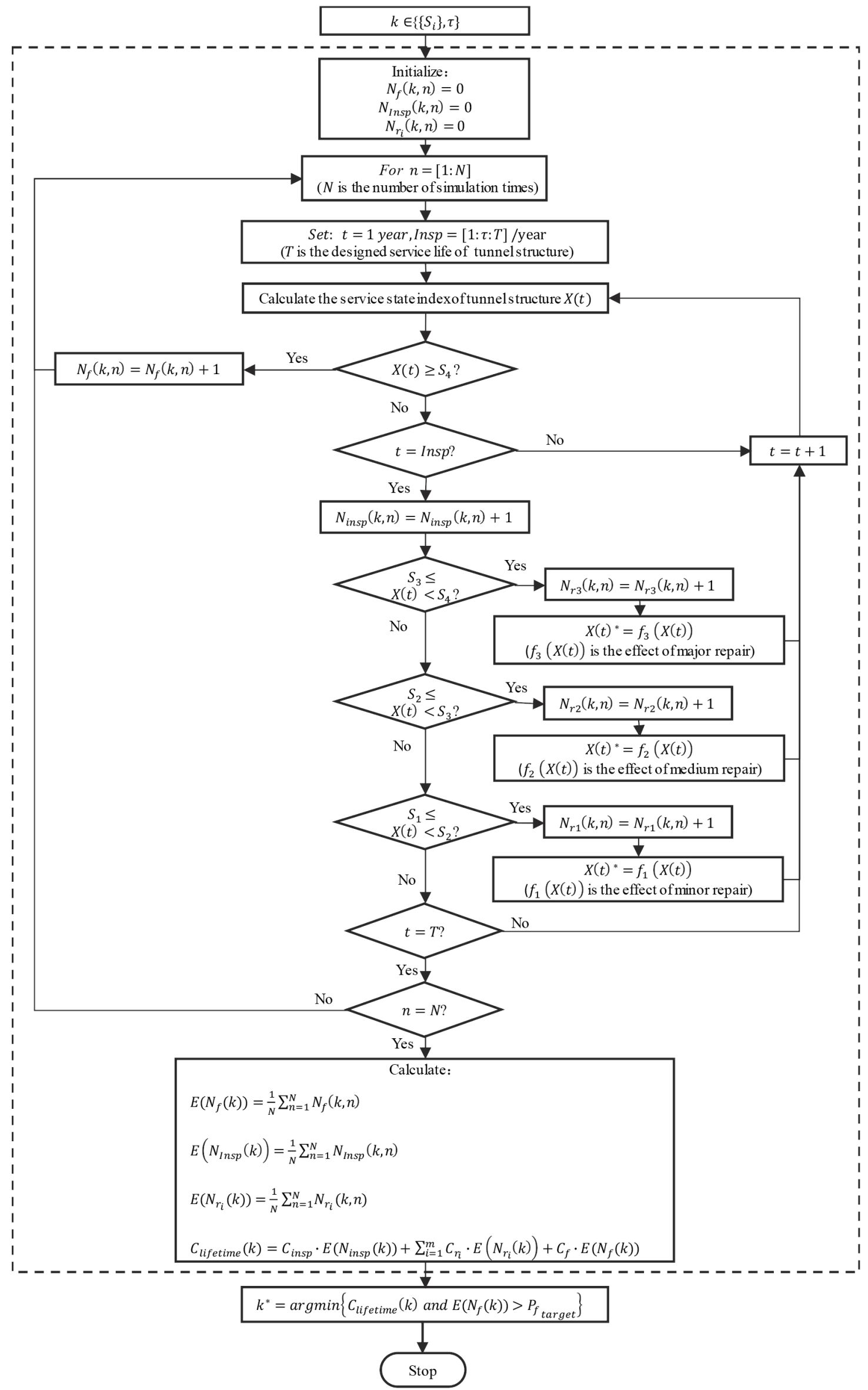

Figure 6. Nested enumeration algorithm for SOM approach (MS-1 as an example) 


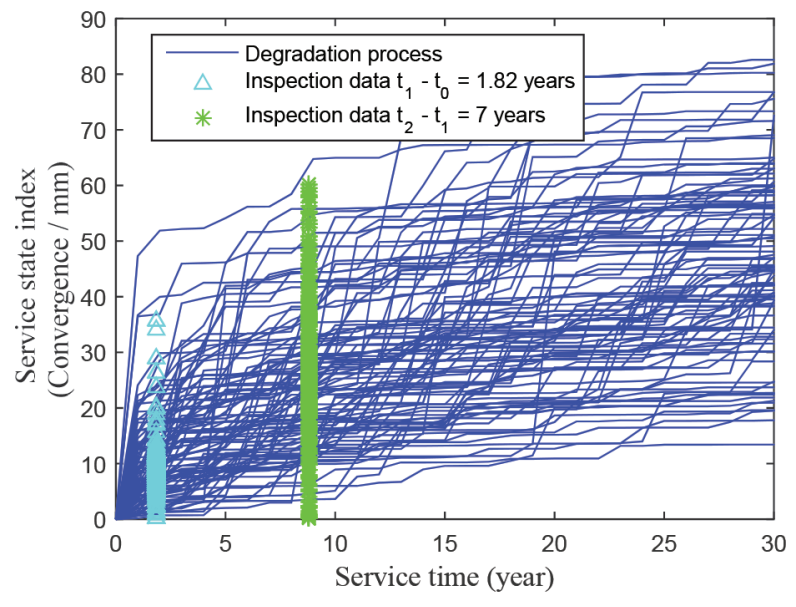

Figure 7. Degradation modelling of investigated tunnel structure

parameter is $\beta$. Its parameters are estimated by the maximum likelihood method based on the inspection data. Table 4 shows the statistics of the increments of convergence for different time periods during service life. The data is collected from 823 segmental rings of the investigated tunnel (Ye 2007).

It should be noted that $t_{0}$ is the time when the tunnel started operation. By Eqn (11), the estimates of the parameters are obtained as $c=1.3211, q=0.5063$ and $\beta=0.1578$. The simulation of investigated tunnel structure is shown in Figure 7. It is observed that the monitoring data mostly falls within the ranges of degradation processes, implying that the degradation modelling is reasonable.

\subsection{Assumptions of maintenance strategy}

Based on the above research, the effects of different degrees of repairing measures (minor repair, medium repair and major repair) are assumed, as shown in Table 5. Note that the minor repair is assumed as the reduction of degradation rate; medium and major repairs are simulated as different reduction of service state index.

The cost of maintenance measure is also important question. Considering the complexity of maintenance measures, usually the assumptions of maintenance costs are connected with the degrees of maintenance measures. Table 6 lists the costs of failure and different repairing measures. The cost of preventive repair in two-levelled service state is the same with major repair in multi-levelled service state in SOM approach.

Table 6. Assumptions of the costs of different maintenance activities

\begin{tabular}{|l|l|}
\hline \multicolumn{1}{|c|}{ Maintenance measures } & \multicolumn{1}{c|}{ Cost } \\
\hline Inspection & 1 \\
\hline Minor repair & 5 \\
\hline Medium repair & 200 \\
\hline Major repair (= preventive repair) & 500 \\
\hline Rehabilitation & 500000 \\
\hline
\end{tabular}

Table 7. Strategies to be optimized and ranges for their parameters

\begin{tabular}{|c|c|c|}
\hline Maintenance strategy & MS-1 & MS-2 \\
\hline $\begin{array}{l}\text { Ranges for } \\
\text { maintenance } \\
\text { strategy parameters }\end{array}$ & $\begin{array}{c}\tau \in[1,40] \\
S_{1}, S_{2}, S_{3} \in[20,140] \\
\left(S_{1}+40 \leq S_{2}+20 \leq S_{3}\right)\end{array}$ & $\begin{array}{r}\tau \in[1,40] \\
S \in[80,140\end{array}$ \\
\hline
\end{tabular}

In general, the threshold of whether a structure enters a Dangerous state (Failure) is known and can be learned from previous experiments or calculations. Therefore, the parameters that need to be optimized in maintenance strategies are the inspection interval and thresholds for different service states. Their ranges for searching the optimal values are shown in Table 7.

\subsection{Results of numerical investigation}

In maintenance strategy MS-1 and MS-2, the inspection interval and thresholds of different service states $S_{i} / S$ are optimized simultaneously. Due to the adoption of SOM approach, there are many parameters to

Table 5. Assumptions of different degrees of repairing measures

\begin{tabular}{|c|c|c|c|}
\hline \multicolumn{2}{|c|}{ Multi-levelled service state } & \multicolumn{2}{c|}{ Two-levelled service state } \\
\hline Degree & Assumption & Degree & Assumption \\
\hline \multirow{3}{*}{ Minor repair } & Reducing degradation rate: & & \\
& $\alpha^{*}=\alpha \cdot d[i]$ & & \\
\hline \multirow{4}{*}{ Medium repair } & Improving service state index: & & \\
& $A(t) \sim U\left(a_{1}, b_{1}\right)$ & Preventive repair & Improving service state index: \\
& $a_{1}=20, b_{1}=40$ & & $A(t) \sim U\left(a_{2}, b_{2}\right)$ \\
Major repair & Improving service state index: & & $a_{2}=40, b_{2}=60$ \\
& $A(t) \sim U\left(a_{2}, b_{2}\right)$ & & \\
& $a_{2}=40, b_{2}=60$ & & \\
\hline
\end{tabular}




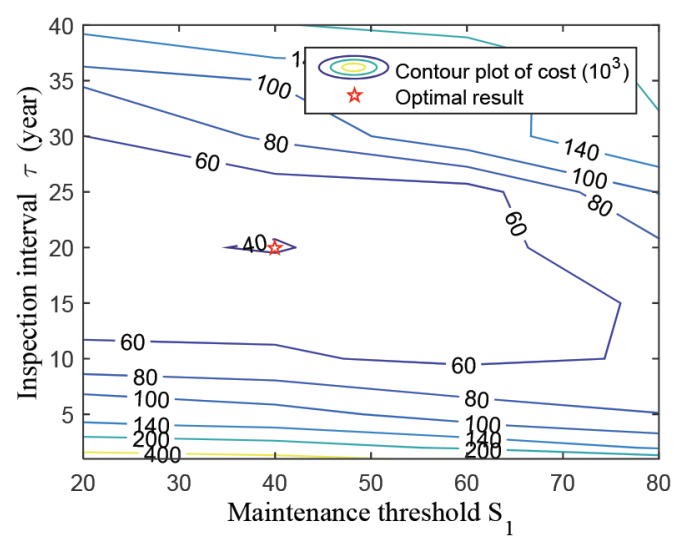

(a) MS-1

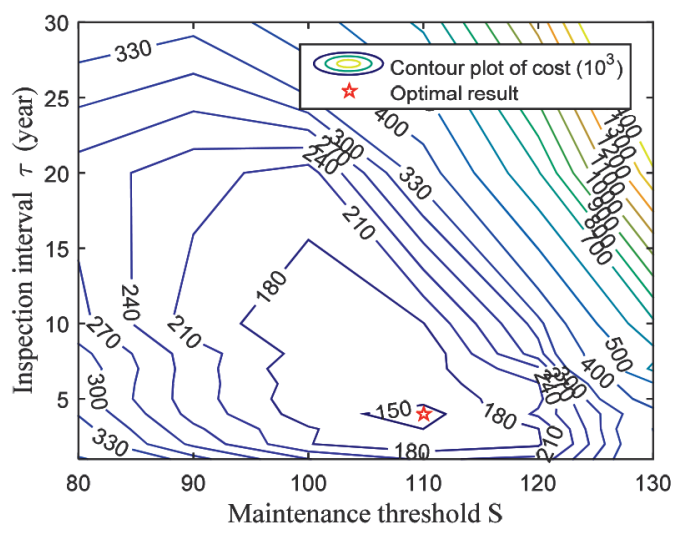

(b) MS-2

Figure 8. Comparison of maintenance costs by contour plot

be optimized in MS-1, and its optimal solution needs to be calculated through multi-dimensional search. In contrast, MS-2 only has two parameters, and it needs less computation than MS-1.

Through the analysis of calculation results in global domain, it is found that MS- 1 has lower maintenance cost with lower failure probability under the combinations of \{ $\left.S_{2}=120, S_{3}=140\right\},\left\{S_{2}=100, S_{3}=140\right\}$, or $\left\{S_{2}=100\right.$, and $\left.S_{3}=120\right\}$. Thus, it is not necessary to focus on the impact of $S_{2}$ and $S_{3}$ because they are not sensitive parameters. Figure 8(a) shows the contour map of maintenance costs of MS-1 varying with parameters $\tau$ and $S_{1}$, in the case of $S_{2}=100, S_{3}=120$. In this combination of parameters, the optimal location is marked as a star in the contour plot. As a comparison, Figure 8(b) shows the contour plot of maintenance cost of MS-2, and the optimal parameters for this strategy are $\tau=4$ and $S=110$.

According to the density features of contour plot in Figure 8 , the maintenance cost of the two maintenance strategies is more sensitive to inspection interval . In order to compare the effects of MS-1 and MS-2 from a more directly view, the maintenance costs varying with different inspection intervals of the two under their optimal maintenance thresholds are shown in Figure 9. It can be observed that the lifetime cost of MS-1 is much lower than MS-2, which indicates that MS-1 is better than MS-2 in most conditions.

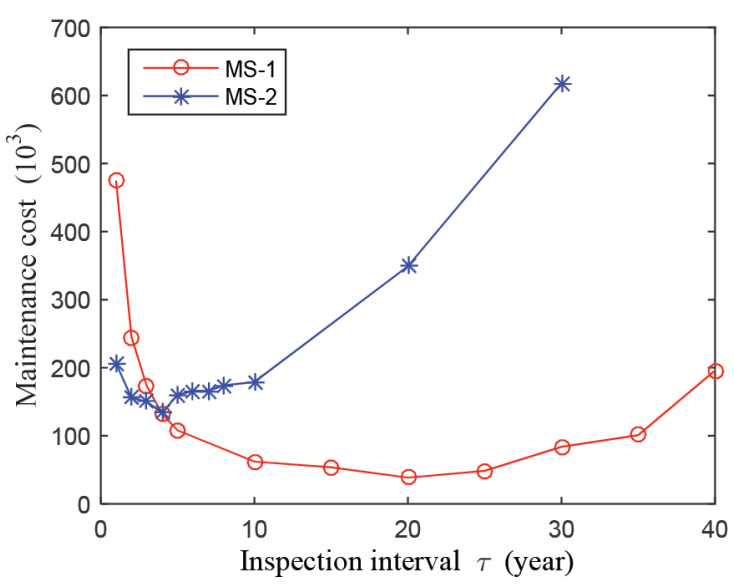

Figure 9. Comparison of MS-1 and MS-2 under the same inspection interval

Considering classification of service state, inspection, lifetime maintenance cost and probability of failure, the results of two maintenance strategies are comprehensively compared in Table 8.

MS-1 sets the service state as a multi-levelled criterion for choosing different degrees of repairing measures, which represents a refined maintenance method. On the other hand, MS-2 adopts two-levelled service state only consisting of safe and failure, so it can be regarded

Table 8. Optimal parameters and maintenance cost for different maintenance strategies

\begin{tabular}{|l|c|c|}
\hline \multicolumn{1}{|c|}{ Maintenance strategy No. } & MS-1 & MS-2 \\
\hline Classification of service state & Multi-levelled service state & Two-levelled service state \\
\hline Inspection type & Periodic inspection & Periodic inspection \\
\hline \multirow{2}{*}{ Optimal parameters } & $\tau=20$ & $\tau=4$ \\
& $S_{1}=40, S_{2}=100, S_{3}=120$ & $S=110$ \\
\hline Optimal maintenance cost & 38.5 & 134 \\
\hline Pf & $5.0 \times 10^{-6}$ & $7.5 \times 10^{-5}$ \\
\hline
\end{tabular}


as an extensive maintenance method. Their difference is that MS-1 has different degrees of repairing activities to choose. Thus, the results of numerical investigation show that when there are different degrees of repairing measures available and minor or medium repair is more effective/cheaper in SOM approach, the maintenance strategy adopting multi-levelled services state will greatly reduce the maintenance cost during the whole service life of tunnel structure.

\section{Conclusions}

This paper proposes a SOM approach to investigate the efficiency of maintenance strategies. SOM approach takes the level of service state as criteria for selecting repairing measures. Two representative maintenance strategies, which are multi-levelled service state and two-levelled service state, are compared in the view of lifetime maintenance cost. The numerical investigations of the two maintenance strategies show that:

1. SOM approach is able to deal with maintenance strategies adopting different classifications of service state, and their effects can be compared by optimization algorithms in the view of lifetime maintenance cost.

2. Minor or medium repairing measures implemented in the early deterioration stage of tunnel will postpone more severe damages happen and reduces lifetime maintenance cost.

3. The maintenance strategy adopting multi-levelled service state (MS-1) can greatly reduce maintenance cost, which is better than MS-2.

Although there are still several limitations, such as the idealized degradation assumption of Gamma process and negligence of extreme effects, SOM approach is validated to be an effective method for allocating resources in the maintenance of tunnel.

\section{Funding}

This work was supported by the China Postdoctoral Science Foundation under Grant number 2017M621474.

\section{Disclosure Statement}

No potential conflict of interest was reported by the authors.

\section{References}

Ai, Q.; Yuan, Y.; Mahadevan, S.; Jiang, X. 2016. Probabilistic degradation modelling of circular tunnels assembled from segmental linings, Structural Concrete 17(2): 257-273. https://doi.org/10.1002/suco.201400122

Baji, H.; Li, C.; Scicluna, S.; Dauth, J. 2017. Risk-cost optimised maintenance strategy for tunnel structures, Tunnelling and Underground Space Technology 69: 72-84. https://doi.org/10.1016/j.tust.2017.06.008

Barker, C. T.; Newby, M. J. 2009. Optimal non-periodic inspection for a multivariate degradation model, Reliability Engi- neering \& System Safety 94(1): 33-43.

https://doi.org/10.1016/j.ress.2007.03.015

Castanier, B.; Grall, A.; Bérenguer, C. 2005. A condition-based maintenance policy with non-periodic inspections for a twounit series system, Reliability Engineering \& System Safety 87(1): 109-120. https://doi.org/10.1016/j.ress.2004.04.013

Chen, C. J.; Juan, Y. K.; Hsu, Y. H. 2017. Developing a systematic approach to evaluate and predict building service life, Journal of Civil Engineering and Management 23(7): 890-901. https://doi.org/10.3846/13923730.2017.1341956

Do, P.; Voisin, A.; Levrat, E.; Iung, B. 2015. A proactive condition-based maintenance strategy with both perfect and imperfect maintenance actions, Reliability Engineering \& System Safety 133: 22-32. https://doi.org/10.1016/j.ress.2014.08.011

Farran, M.; Zayed, T. 2015. Fitness-oriented multi-objective optimisation for infrastructures rehabilitations, Structure and Infrastructure Engineering 11(6): 761-775. https://doi.org/10.1080/15732479.2014.905964

GB50153-2008 Unified standard for reliability design of engineering structures. Beijing: China Architecture and Building Press, 2008 (in Chinese).

Grall, A.; Bérenguer, C.; Dieulle, L. 2002. A condition-based maintenance policy for stochastically deteriorating systems, Reliability Engineering \& System Safety 76(2): 167-180. https://doi.org/10.1016/S0951-8320(01)00148-X

Jardine, A. K.; Lin, D.; Banjevic, D. 2006. A review on machinery diagnostics and prognostics implementing condition-based maintenance, Mechanical Systems and Signal Processing 20(7): 1483-1510. https://doi.org/10.1016/j.ymssp.2005.09.012

Jin, Y-F.; Yin, Z-Y.; Shen, S-L.; Hicher, P-Y. 2016. Selection of sand models and identification of parameters using an enhanced genetic algorithm, International Journal for Numerical and Analytical Methods in Geomechanics 40(8): 1219-1240. https://doi.org/10.1002/nag.2487

Jin, Y.-F.; Yin, Z.-Y.; Shen, S.-L.; Zhang, D.-M. 2017. A new hybrid real-coded genetic algorithm and its application to parameters identification of soils, Inverse Problems in Science and Engineering 25(9): 1343-1366.

https://doi.org/10.1080/17415977.2016.1259315

JTG H12-2015 Highway tunnel maintenance technical specifications. Beijing: People's Communication Publishing House Co., Ltd., 2015 (in Chinese).

Kavussi, A.; Abbasghorbani, M.; Moghadas Nejad, F.; Bamdad Ziksari, A. 2017. A new method to determine maintenance and repair activities at network-level pavement management using falling weight deflectometer, Journal of Civil Engineering and Management 23(3): 338-346. https://doi.org/10.3846/13923730.2015.1073173

Li, X.; Lin, X.; Zhu, H.; Wang, X.; Liu, Z. 2017. Condition assessment of shield tunnel using a new indicator: The tunnel serviceability index, Tunnelling and Underground Space Technology 67: 98-106. https://doi.org/10.1016/j.tust.2017.05.007

Liu, H.; Madanat, S. 2015. Adaptive optimisation methods in system-level bridge management, Structure and Infrastructure Engineering 11(7): 884-896. https://doi.org/10.1080/15732479.2014.920038

Lyu, H. M.; Shen, J. S.; Arulrajah, A. 2018. Assessment of geohazards and preventative countermeasures using AHP incorporated with GIS in Lanzhou, China, Sustainability 10(2): 304. https://doi.org/10.3390/su10020304

Marzouk, M.; Omar, M. 2013. Multiobjective optimisation algorithm for sewer network rehabilitation, Structure and Infrastructure Engineering 9(11): 1094-1102. https://doi.org/10.1080/15732479.2012.666254 
Neves, M. L.; Santiago, L. P.; Maia, C. A. 2011. A condition-based maintenance policy and input parameters estimation for deteriorating systems under periodic inspection, Computers \& Industrial Engineering 61(3): 503-511. https://doi.org/10.1016/j.cie.2011.04.005

Nicolai, R. P.; Dekker, R.; Van Noortwijk, J. M. 2007. A comparison of models for measurable deterioration: An application to coatings on steel structures, Reliability Engineering \& System Safety 92(12): 1635-1650. https://doi.org/10.1016/j.ress.2006.09.021

Nicolai, R. P.; Frenk, J. B. G.; Dekker, R. 2009. Modelling and optimizing imperfect maintenance of coatings on steel structures, Structural Safety 31(3): 234-244.

https://doi.org/10.1016/j.strusafe.2008.06.015

Ponchet, A.; Fouladirad, M.; Grall, A. 2012. Imperfect conditionbased maintenance assessment on a finite time span, in 2012 International Conference on Quality, Reliability, Risk, Maintenance, and Safety Engineering (ICQR2MSE), 2012, 390-395. https://doi.org/10.1109/ICQR2MSE.2012.6246260

Strauss, A.; Wan-Wendner, R.; Vidovic, A.; Zambon, I.; Yu, Q.; Frangopol, D. M.; Bergmeister, K. 2017. Gamma prediction models for long-term creep deformations of prestressed concrete bridges, Journal of Civil Engineering and Management 23(6): 681-698. https://doi.org/10.3846/13923730.2017.1335652

Van Noortwijk, J. M. 2009. A survey of the application of gamma processes in maintenance, Reliability Engineering \& System Safety 94(1): 2-21. https://doi.org/10.1016/j.ress.2007.03.019

Van, P. D.; Bérenguer, C. 2012. Condition-based maintenance with imperfect preventive repairs for a deteriorating production system, Quality and Reliability Engineering International 28(6): 624-633. https://doi.org/10.1002/qre.1431

Wang, J.; Koizumi, A.; Tanaka, H. 2017. Framework for maintenance management of shield tunnel using structural performance and life cycle cost as indicators, Structure and Infrastructure Engineering 13(1): 44-54. https://doi.org/10.1080/15732479.2016.1198406

Ye, Y. D. 2007. Research on deformation and method of health diagnose of operational subway structures in soft soil: Doctoral Thesis. Shanghai: Tongji University.

Yin, Z. Y.; Jin, Y. F.; Shen, J. S.; Hicher, P. Y. 2018. Optimization techniques for identifying soil parameters in geotechnical engineering: Comparative study and enhancement, International Journal for Numerical and Analytical Methods in Geomechanics 42: 70-94. https://doi.org/10.1002/nag.2714

Yuan, Y.; Bai, Y.; Liu, J. 2012. Assessment service state of tunnel structure, Tunnelling and Underground Space Technology 27(1): 72-85. https://doi.org/10.1016/j.tust.2011.07.002

Zambon, I.; Vidovic, A.; Strauss, A.; Matos, J.; Amado, J. 2017. Comparison of stochastic prediction models based on visual inspections of bridge decks, Journal of Civil Engineering and Management 23(5): 553-561. https://doi.org/10.3846/13923730.2017.1323795

Zhang, W.; Sun, K.; Lei, C.; Zhang, Y.; Li, H.; Spencer, B. F. 2014. Fuzzy analytic hierarchy process synthetic evaluation models for the health monitoring of shield tunnels, Computer-Aided Civil and Infrastructure Engineering 29(9): 676-688. https://doi.org/10.1111/mice.12091

Zhu, Q.; Peng, H.; van Houtum, G. J. 2015. A condition-based maintenance policy for multi-component systems with a high maintenance setup cost, OR Spectrum 37(4): 1007-1035. https://doi.org/10.1007/s00291-015-0405-z 